\title{
A Growth Chart for Premature and Other Infants
}

\author{
DOUGLAS GAIRDNER and JULIE PEARSON \\ From Addenbrooke's Hospital, Cambridge, and Cambridge Maternity Hospital
}

\begin{abstract}
Gairdner, D., and Pearson, J. (1971). Archives of Disease in Childhood, 46, 783. A growth chart for premature and other infants. The growth chart covers the period from 28 weeks of fetal life to age 2 years, and allows weight, length, and head circumference to be charted. The time scale is logarithmic, providing the convenience of an extended scale for the earlier months of life, and giving the graphs an approximately linear form. Separate charts for boys and girls are provided, with 10th, 50th, and 90th centiles.

Weight charts of individual low birthweight infants illustrate the striking 'catch-up' growth often achieved by these infants.
\end{abstract}

These charts (Fig. 1 and 2) $\dagger$ have been designed to enable the paediatrician to chart the course of growth in the early months of life as meaningfully as possible. They incorporate two features. Firstly, in order to accommodate those infants born preterm, curves for normal growth in the period 28 to 40 weeks' gestational age are shown as continuous with those for later ages. Secondly, the time scale is logarithmic, so that the scale is extended for the early weeks of life, the period when growth is rapid.

The sources of the composite data from which the curves are derived are as follows:

(1) Weight and length from birth at 40 weeks' gestation to 2 years, from the British data of Tanner, Whitehouse, and Takaishi (1966).

(2) Head circumference from birth at 40 weeks' gestation to 2 years, from the Harvard data published by Nelson (1964).

(3) Weight from 32 to 40 weeks' gestation, from the curves given by Tanner and Thomson (1970) for Aberdeen infants. These authors provide separate curves for first-born and later-born infants, and the data for later-born infants have been used here.

(4) Weight at 28 weeks' gestation, from the composite data of Babson (1970). His data combine both sexes and are given in terms of mean and standard deviation (SD). By multiplying the SD by a factor of $1 \cdot 28$, the

Received 11 June 1971.

tThe chart measures $28 \mathrm{~cm}$ in height, to match the usual size of hospital records. Breadth is $30 \mathrm{~cm}$, including a wide margin for binding; a single vertical fold enables the chart to be incorporated in a patient's records. Supplied by Creaseys, Bull Plain, Hertford, Herts. corresponding 10th and 90th centiles were derived. Curves were then drawn from these points to meet the weight curves at 32 weeks.

(5) Length and head circumference from 28 to 40 weeks' gestation, from the data of Babson (1970), which combine both sexes; 10 th and 90 th centiles were derived as above from the $\mathrm{SD}$ values.

Despite the variety of sources of the data, no major problems arose in combining them, and the composite curves had only to be slightly smoothed.

One convenience of plotting parameters of growth against a logarithmic time scale is that the graphs then tend to become linear, owing to the roughly exponential fall-off of growth rates during the period covered by the charts. With the time scale adopted in the charts, this applies particularly to the graphs for weight and length; the graph for head circumference remains somewhat curved because of the relatively more rapid decline in the rate of growth of the head.

It is generally accepted that the fetus gains weight at a slower rate during the last weeks of pregnancy, though the extent of the decline varies in fetal growth curves as constructed by different authors (see Babson, Behrman, and Lessel, 1970). To what extent growth in length and in head circumference similarly declines is less well documented, but Usher and McLean's (1969) data suggest that the effect is not so marked for these parameters. (In the chart for girls (Fig. 2), the apparent decline in rate of growth of the head shown at 38 weeks may well be due to insufficient smoothing of this curve: slight modification of this part of the curve has been made in the current version of the chart.) 


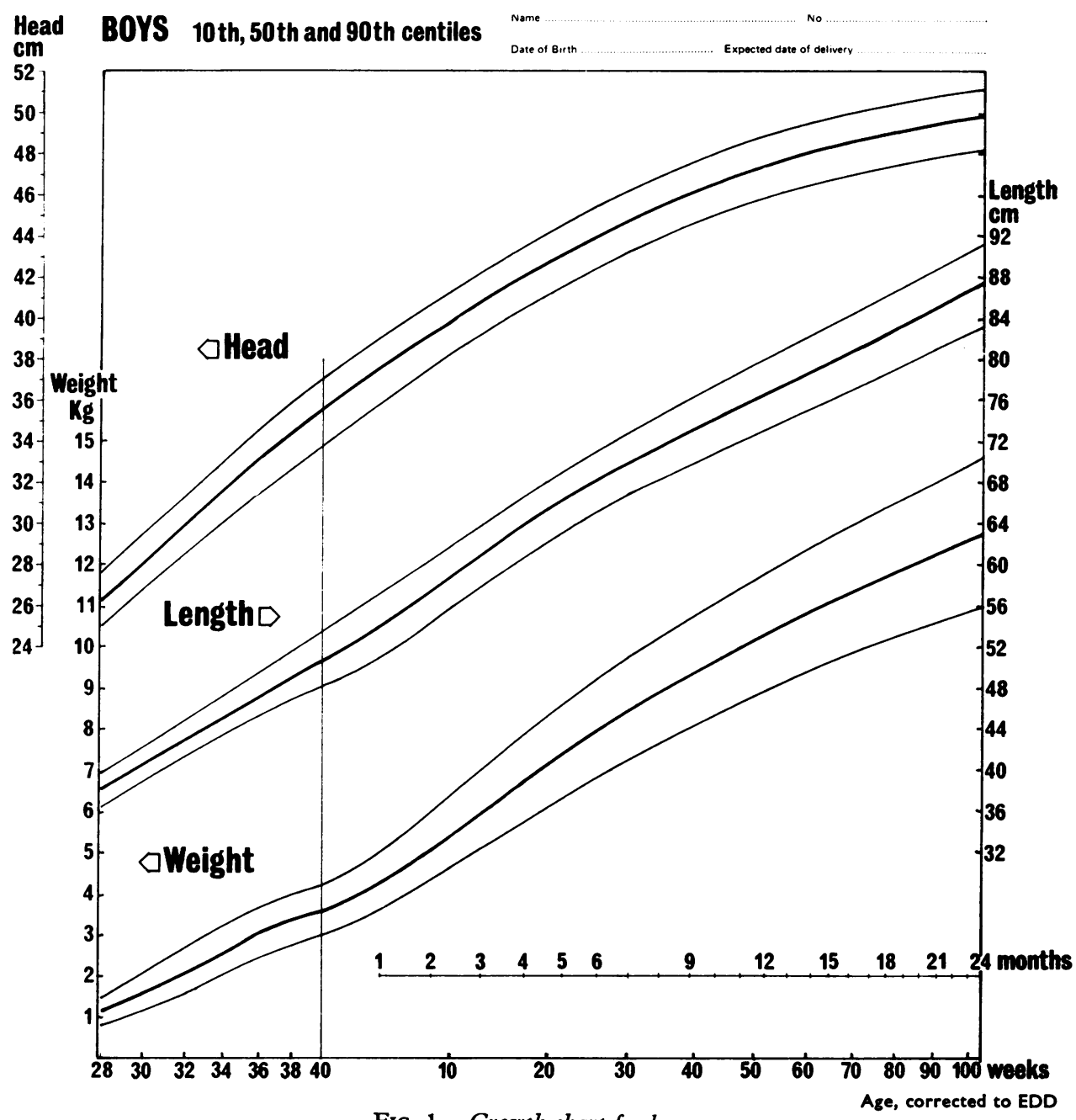

FIG. 1.-Growth chart for boys.

Taking the data of Tanner and Thomson, the daily weight gain of the average (male) fetus over the last two months of gestation, is $36 \mathrm{~g} /$ day between 32 and 36 weeks, falling to $25 \mathrm{~g} /$ day between 36 and 38 weeks, and then to $14 \mathrm{~g} /$ day between 38 and 40 weeks. After 40 weeks, weight gains become negligible, $3.5 \mathrm{~g} /$ day between 40 and 42 weeks.

That this decline in fetal growth rate after about 36 weeks is imposed on the fetus by maternal (uterine and placental) factors, is proved by the fact that infants who successfully make their escape from the confines of the uterus before term show no such fall-off in growth rate (see Fig. 3 and 4). It has to be accepted, therefore, that graphs of normal fetal growth can never provide a standard wholly appropriate to the extrauterine growth to be expected of an infant delivered before term. When a premature infant reaches the gestational age of about 36 weeks, he will usually be seen to grow much faster than does the fetus (see Fig. 3, 4, and 5), to the extent that if he does not, the possibility of some abnormality in the infant or his feeding should be considered.

\section{Correction of Age for Infants born Prematurely}

The vertical line on the chart corresponding to 40 weeks' gestation represents the expected date of delivery (EDD), 280 days after the start of the 


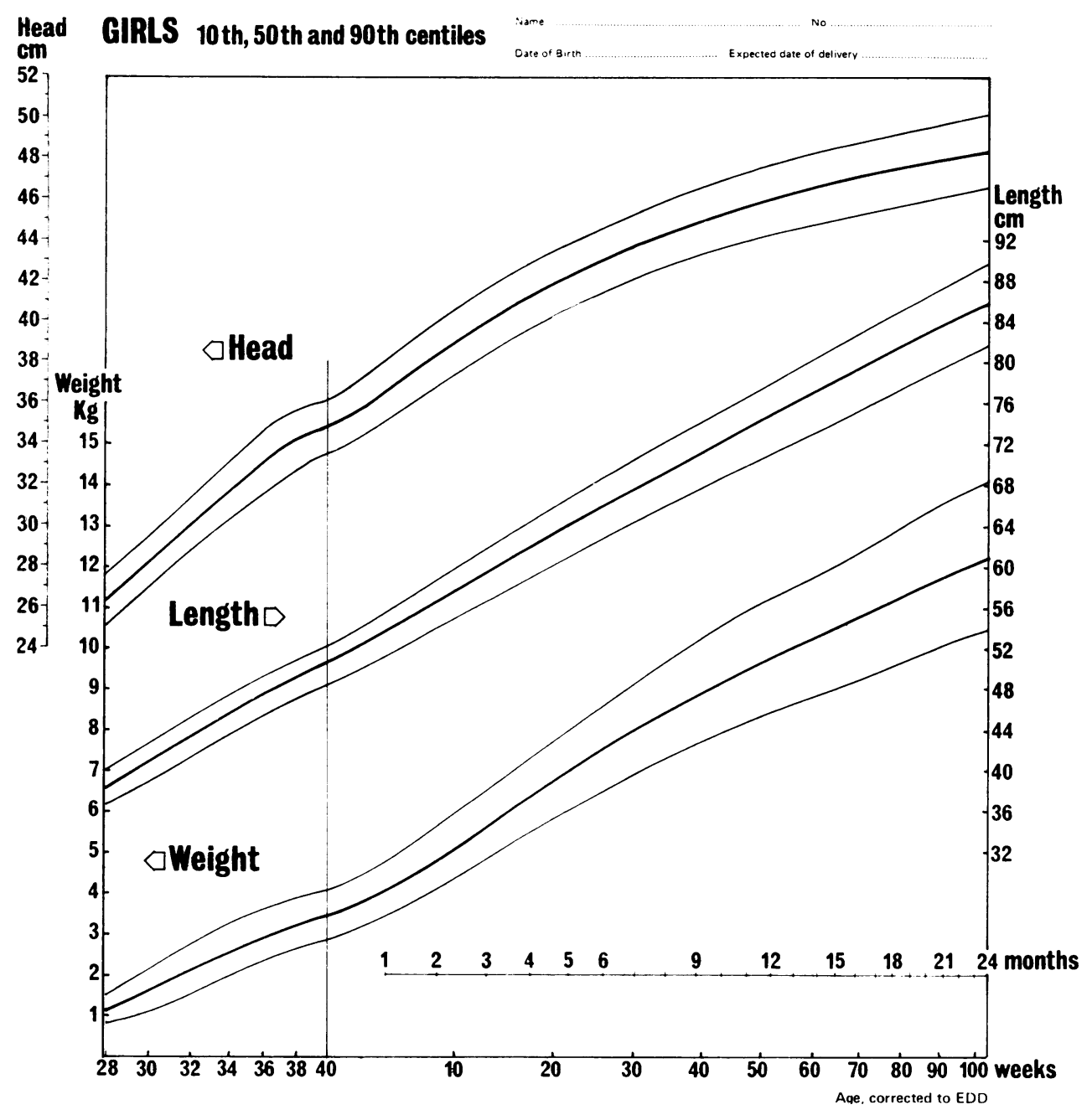

FIG. 2.-Growth chart for girls.

last menstrual period, so that the postnatal age scale shown to the right of this line applies, if uncorrected, to infants born at term. For a premature infant born $n$ weeks before term, correction of postnatal age is made, either by subtracting $n$ weeks from the postnatal age, or alternatively by taking the EDD as the effective date of birth.

No analogous correction of postnatal age need be made for an infant born postterm, since fetal growth after 40 weeks is negligible (see above).

\section{Growth of Premature Infants}

By way of illustrating the use of the charts, some of the commoner patterns of weight gain to be seen in two groups of infants, premature and 'small-fordates', may be considered.

Fig. 3 shows the weight chart of a premature infant born at 28 weeks. In a thriving premature infant such as this, four phases of growth are typically to be observed.

Phase 1: the immediate postnatal weight loss.

Phase 2: starting $\frac{1}{2}$ to 1 week after birth, the curve runs parallel to the fetal weight curve (usually below it). The rate of growth is thus similar to that of the fetus of like gestational age. This phase lasts for from 2 to 5 weeks, the longer period applying to the smallest infants.

Phase 3: growth now accelerates, reaching a velocity that usually much exceeds that of the fetus 


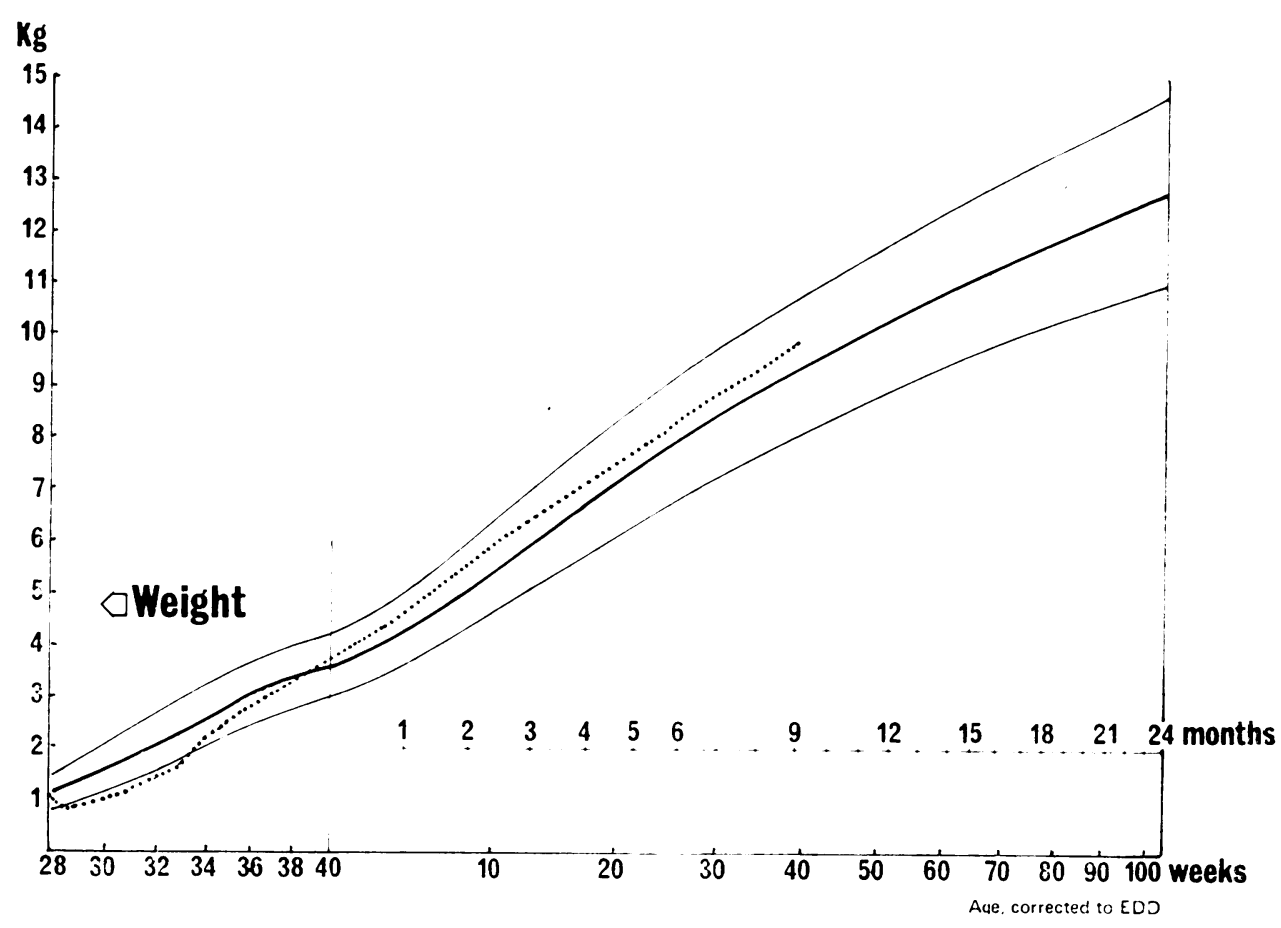

FIG. 3.-Weight chart of a healthy premature (male) infant, weight $1.1 \mathrm{~kg}$ at 28 weeks. After the initial weight loss, growth at first runs parallel to the fetal weight curve (29-33 weeks), after which there is a phase of rapid growth, leading to a normal weight at the 'expected date of delivery'.

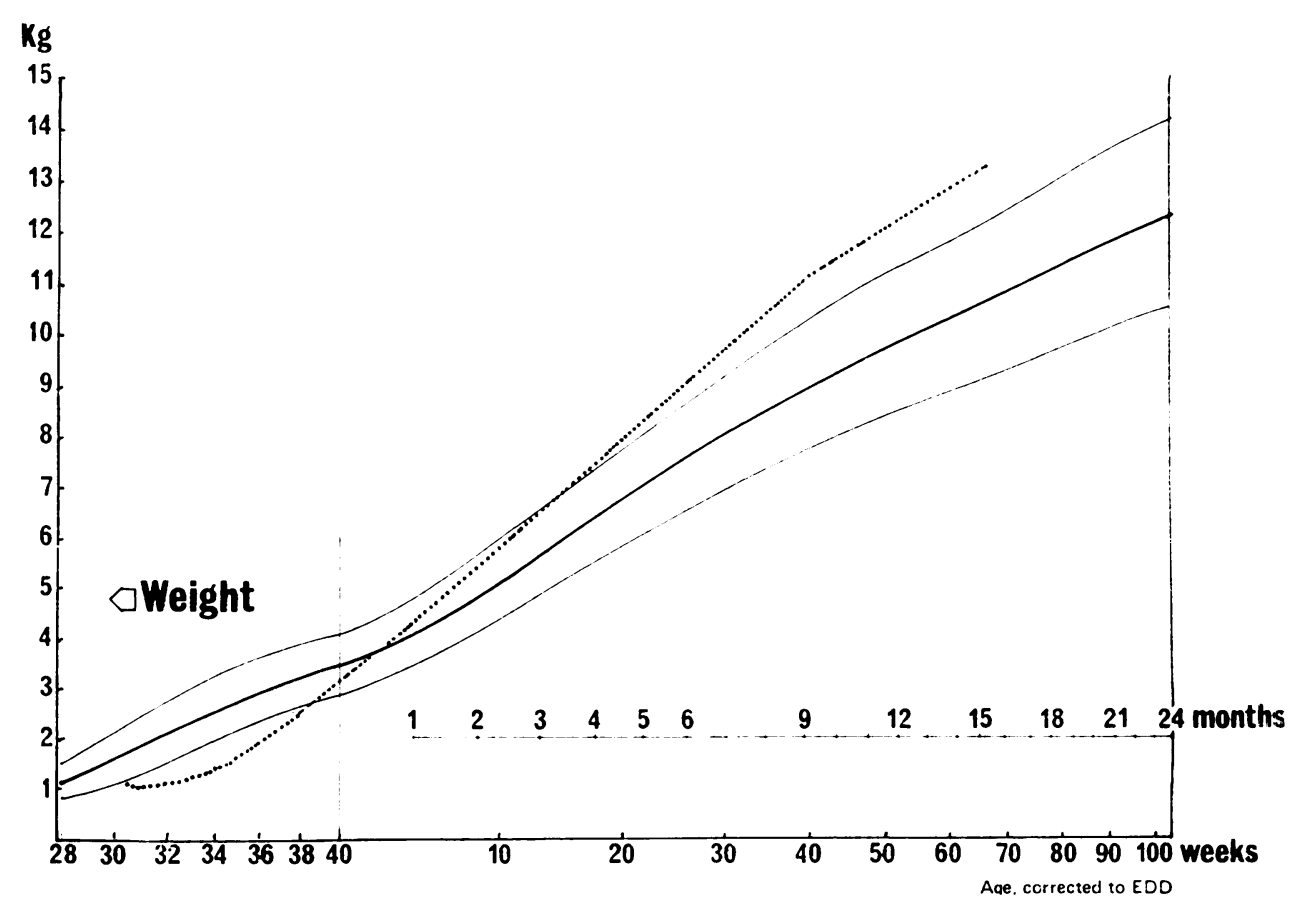

Fig. 4.-Development of obesity in a premature (female) infant, weight $1.1 \mathrm{~kg}$ at $30 \frac{1}{2}$ weeks' gestation. After a 'physiological' phase of accelerated growth from 35 weeks' gestational age, there was a persistence of rapid weight gain, which led to obvious obesity after the (corrected) age of 4 months. 


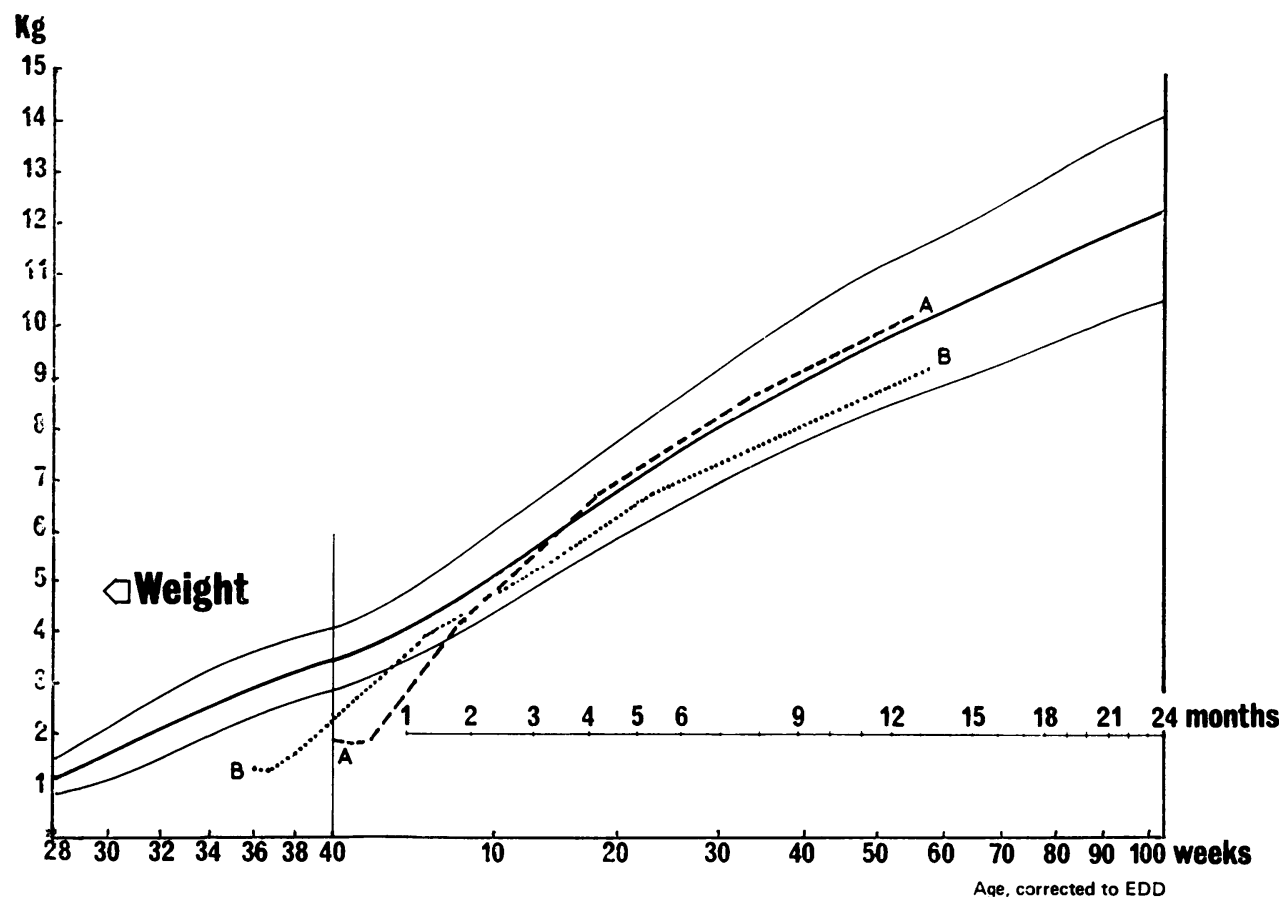

FIG. 5.-'Catch-up' growth in two 'small-for-dates' (female) infants; A born at term, B at 36 weeks' gestation (see text).

of comparable gestational age, often surpassing the rate of $36 \mathrm{~g} /$ day at which the fetus grows during the period of its most rapid growth. Thus the infant cited in Fig. 3 sustained a growth rate of $41 \mathrm{~g} /$ day during the 7 weeks from 33 to 40 weeks gestational age.

This rapid growth is usually maintained by the infant until he reaches what is presumably his genetically appropriate weight centile, after which (Phase 4) growth tends to continue along the same centile, as in Fig. 3. However, it is also not uncommon for a premature infant to grow decidedly obese, continuing to put on weight at a rate which causes the weight curve to cross the centiles, as in Fig. 4.

\section{Growth of 'Small-for-dates' Infants}

Fig. 5 illustrates the growth of two 'small-fordates' infants, one born at term and the other preterm, and both showing impressive postnatal 'catchup' growth. Infant $A$ was born at term and weighed $1.9 \mathrm{~kg}$ or $54 \%$ of the expected weight; the accelerated growth phase lasted for 7 weeks, after which the weight curve continued just above the 50th centile. Infant B was born at 36 weeks' gestation and weighed $1.3 \mathrm{~kg}$ or $45 \%$ of expected weight. The accelerated growth phase lasted 9 weeks; at a (corrected) postnatal age of 5 weeks the weight curve flattened, and continued thereafter between the 10th and 50th centiles. In both cases, the rate of growth was $43 \mathrm{~g} /$ day, compared with the $24 \mathrm{~g} /$ day gained by the average infant between the ages of 2 and 8 weeks.

We are greatly indebted to Dr. Gillian Gandy who suggested the usefulness of a logarithmic time scale.

\section{REFERENCES}

Babson, S. G. (1970). Growth of low-birthweight infants. Fournal of Pediatrics, 77, 11.

Babson, S. G., Behrman, R. E., and Lessel, R. (1970). Fetal growth: liveborn birth weights for gestational age of white middle class infants. Pediatrics, 45, 937.

Nelson, W. E. (1964). Textbook of Pediatrics, 8th ed., p. 48. W. B. Saunders, Philadelphia.

Tanner, J. M., and Thomson, A. M. (1970). Standards of birthweight at gestation periods from 32 to 42 weeks, allowing for maternal height and weight. Archives of Disease in Childhood, 45, 566.

Tanner, J. M., Whitehouse, R. H., and Takaishi, M. (1966). Standards from birth to maturity for height, weight, height velocity, and weight velocity: British children, 1965. Archives of Disease in Childhood, 41, 454, 613.

Usher, R., and McLean, F. (1969). Intrauterine growth of liveborn Caucasian infants at sea level: standards obtained from measurements in 7 dimensions of infants born between 25 and 44 weeks of gestation. Fournal of Pediatrics, 74, 901.

Correspondence to Dr. D. Gairdner, Addenbrooke's Hospital, Cambridge. See footnote on p. 783 for supplies of charts. 